\title{
The cotton GhWIN2 gene activates the cuticle biosynthesis pathway and influences the salicylic and jasmonic acid biosynthesis pathways
}

Xiancai Li ${ }^{1}$, Nana Liu', Yun Sun ${ }^{1}$, Ping Wang ${ }^{1}$, Xiaoyang Ge ${ }^{2}$, Yakun Pei ${ }^{1}$, Di Liu', Xiaowen Ma ${ }^{1}$, Fuguang $\mathrm{Li}^{2 *}$ and Yuxia Hou ${ }^{1 *}$

\begin{abstract}
Background: Metabolic pathways are interconnected and yet relatively independent. Genes involved in metabolic modules are required for the modules to run. Study of the relationships between genes and metabolic modules improves the understanding of metabolic pathways in plants. The WIN transcription factor activates the cuticle biosynthesis pathway and promotes cuticle biosynthesis. The relationship between the WIN transcription factor and other metabolic pathways is unknown. Our aim was to determine the relationships between the main genes involved in cuticle biosynthesis and those involved in other metabolic pathways. We did this by cloning a cotton WIN gene, GhWIN2, and studying its influence on other pathways.

Results: As with other WIN genes, GhWIN2 regulated expression of cuticle biosynthesis-related genes, and promoted cuticle formation. Silencing of GhWIN2 resulted in enhanced resistance to Verticillium dahliae, caused by increased content of salicylic acid (SA). Moreover, silencing of GhWIN2 suppressed expression of jasmonic acid (JA) biosynthesis-related genes and content. GhWIN2 positively regulated the fatty acid biosynthesis pathway upstream of the JA biosynthesis pathway. Silencing of GhWIN2 reduced the content of stearic acid, a JA biosynthesis precursor.
\end{abstract}

Conclusions: GhWIN2 not only regulated the cuticle biosynthesis pathway, but also positively influenced JA biosynthesis and negatively influenced SA biosynthesis.

Keywords: Cuticle, GhWIN2, Jasmonic acid, Salicylic acid, Systems biology, VIGS, Verticillium dahliae

\section{Background}

Plants are constantly stimulated by environmental signals, some of which inhibit growth and development. Plants have developed many structures, such as the cuticle, that increase adaptation or tolerance to these stresses [1,2]. The plant cuticle is a ubiquitous and chemically heterogeneous lipophilic layer composed of biopolymers, mainly comprising waxes, and cutin, a lipid polymer [3]. The cutin matrix consists mainly of esterified 16/18-carbon hydroxy and epoxy-hydroxy fatty acids

\footnotetext{
* Correspondence: aylifug@126.com; yuxiacau@163.com

${ }^{2}$ State Key Laboratory of Cotton Biology, Institute of Cotton Research of the Chinese Academy of Agricultural Sciences, Anyang 455000, China

${ }^{1}$ College of Science, China Agricultural University, No. 2 Yuanmingyuan West Road, Beijing 100193, China
}

(FAs) [4]. The waxes are formed by very-long-chain fatty acid (VLCFA) derivatives [5], and they cover or are embedded in the cutin matrix. The wax components are produced in three steps. First, 16/18-carbon long-chain acyl-coenzymeAs $\left(\mathrm{C}_{16} / \mathrm{C}_{18}\right.$-acyl-CoAs $)$ are produced from 16/18-carbon long-chain FAs that are catalyzed by long-chain acyl-CoA synthetases (LACSs) in the plastids of epidermal cells [6]. Second, the FAs are extended from $\mathrm{C}_{16} / \mathrm{C}_{18}$-acyl-CoAs to VLCFA-acyl-CoAs $\left(>\mathrm{C}_{18}\right.$, with more than $18 \mathrm{C}$ atoms), catalyzed by fatty acid elongases (FAEs) on the endoplasmic reticulum membrane [7]. FAE enzyme complexes consist of $\beta$-ketoacylCoA synthase (KCS), $\beta$-ketoacyl-CoA reductase (KCR), 3-hydroxyacyl-CoA dehydratase (HCD), and enoyl-CoA 
reductase (ECR) [8-12] Finally, VLCFA-acyl-CoAs further react to form wax components, mediating the alcohol- or alkane-forming pathways [7, 13-16]. Many genes encoding enzymes involved in these pathways have been studied.

WIN1 (wax inducer 1) was first reported to transcriptionally activate epidermal wax biosynthesis in Arabidopsis [17]. The barley WIN/SHN gene, Nud, controls grain with adhering hulls by regulating a lipid biosynthesis pathway [18]. The tomato (Solanum lycopersicum) SlWIN3/SHN3 gene regulates cuticle formation in fleshy fruits [19]. WIN transcription factors function redundantly to regulate the epidermal patterning of flower organs in Arabidopsis [13]. Most of the studies about WIN genes have focused on the regulation of cuticle biosynthesis. In addition, overexpression of an Arabidopsis WIN gene in rice activates cellulose biosynthesis and represses lignin biosynthesis [20]. Beyond that, there have been no reports about the influence of WIN on other metabolic pathways.

Jasmonic acid (JA) is an important plant hormone. It is needed for plant growth and development, survival under stress, and throughout the life-cycle. JA is biosynthesized from $\alpha$-linolenic acid [21]. Linolenic acid is catalyzed by lipoxygenase (LOX) to produce 13-hydroperoxyoctadecatrienoic acid (13-HPOT) [22]. Stearic acid is converted to oleic acid, linoleic acid, and further to $\alpha$-linolenic acid through a desaturation reaction [23]. Stearic acid has a role into the cuticle biosynthesis pathway [24]. The JA biosynthesis pathway competes with the cuticle biosynthesis pathway for precursors.

Salicylic acid (SA) is an important hormone that is involved in plant immune responses. It regulates the expression of many pathogenesis-related proteins (PRs) [25]. SA is involved in plant defense against Verticillium dahliae [26-29]. Two pathways of SA biosynthesis in plants have been reported. First, in Arabidopsis, SA appears to be synthesized primarily through an isochorismate-utilizing pathway. Second, phenylalanine forms a substrate in the SA biosynthesis pathway [30]. Both pathways begin with shikimic acid. Lignin is a phenolic heteropolymer that constitutes an important component of plant secondary cell walls, and shikimic acid is the precursor of lignin biosynthsis [31]. The shikimate pathway is responsible for the biosynthesis of tryptophan, tyrosine, and phenylalanine [32]. Phenylalanine is involved in the lignin pathway. AtWIN1 negatively regulates lignin biosynthesis in transgenic rice plants [20]. The biosynthesis of lignin and SA share part of the same pathway [33]. Therefore, WIN has a role in lignin biosynthesis and may also influence SA accumulation.

Ideker [34] proposed the concept of systems biology in 2001. Briefly, systems biology is the study of living systems not only in terms of separate mechanistic and molecular-level components, but considering many components simultaneously [34, 35]. Metabolic pathways are interconnected, and yet they are relatively independent. For example, various hormone metabolic pathways exert their biological functions synergistically or antagonistically by forming complex and intersecting networks of regulatory pathways [36]. The WIN transcription factor positively regulates the cuticle biosynthesis pathway [17, 37]. Aside from its effects on lignin biosynthesis, it is unknown how the WIN transcription factor influences other metabolic pathways related to the cuticle biosynthesis pathway [20]. Here, we cloned a WIN gene, GhWIN2, from cotton (Gossypium hirsutum), and aimed to determine its role in the regulation of cuticle development and the influence on SA and JA accumulation.

\section{Results}

\section{Characterization of GhWIN2}

Many WIN/SHN orthologs have been reported to affect various aspects in plant physiological processes [17, 37]. However, less is known about the cotton WIN/SHN orthologs. By using the full-length AtWIN1 amino acid sequence to perform a Blast query against the G. hirsutum genome database (https://cottonfgd.org/), we found eleven sequences of WIN/SHN orthologs in the cotton genome. Those sequences encode six putative amino acid sequences (Additional file 1: Figure S1). Next, primers were designed for full length amplification of those SHN orthologs. Unfortunately, we only cloned Gh_A07G036100 (XP_016720718) from two-week-old cotton seedlings. No expression of other homologous sequences was detected by RT-qPCR in seedings. Phylogenetic analysis revealed that this sequence was closely related to SlSHN2/WIN2 (Fig. 1a) [19]. Thus, we named this gene GhWIN2.

We detected subcellular localization in the transgenic Arabidopsis plants that stably expressed GhWIN2 (Additional file 1: Figure S2 and Figure S3). We selected transgenic Arabidopsis line 4, which had the highest expression levels among the transgenic lines, for analyzing the subcellular localization. DAPI staining was used to stain the nucleus. GhWIN2-GFP and DAPI fluorescence were colocalized in the nucleus (Fig. 1b).

To explore the expression pattern of GhWIN2, we analyzed the GhWIN2 promoter sequence obtained from the G. hirsutum genome database (Table 1). This promoter sequence contains cis-acting elements that are involved in ABA and drought response. The expression of GhWIN2 was strongly induced by abscisic acid (ABA) and sodium chloride $(\mathrm{NaCl})$ treatment in cotton seedlings (Fig. 1c and d). 

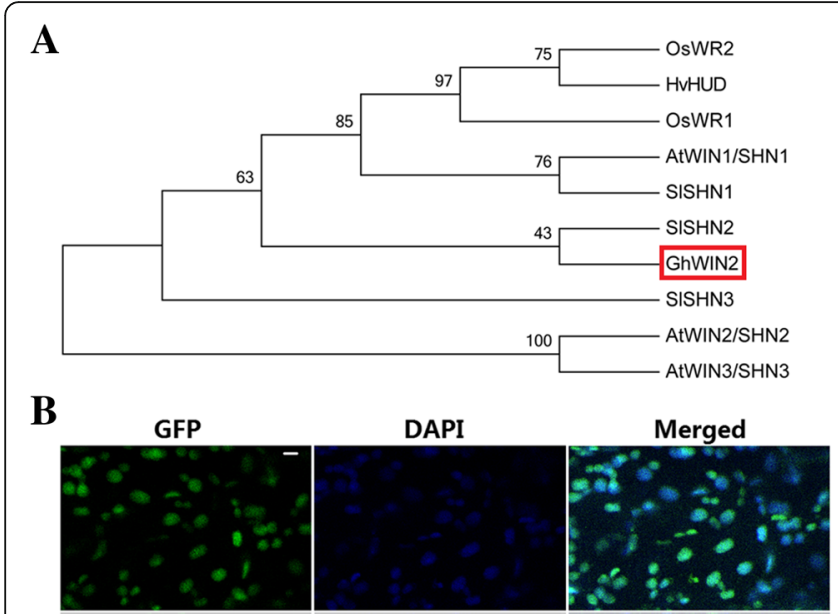

Table 1 The putative cis-acting elements in GhWIN2 promoter

\begin{tabular}{c|c|c|c}
\hline Cis-acting element & Element sequence & Number & Function \\
\hline CBFHV & RYCGAC & 1 & dehydration \\
DRECRTCOREAT & RCCGAC & 1 & drought, salt \\
LTRECOREATCOR15 & CCGAC & 1 & ABA, drought \\
MYB2AT & TAACTG & 1 & dehydration \\
RYREPEATBNNAPA & CATGCA & 1 & ABA \\
MYCATERD1 & CATGTG & 1 & dehydration \\
MYCATRD22 & CACATG & 2 & dehydration, ABA \\
DPBFCOREDCDC3 & ACACNNG & 3 & ABA \\
GT1GMSCAM4 & GAAAAA & 4 & salt \\
MYBCORE & CNGTTR & 5 & dehydration \\
MYB2CONSENSUSAT & YAACKG & 4 & dehydration \\
ACGTATERD1 & ACGT & 2 & dehydration \\
\hline
\end{tabular}

C

D
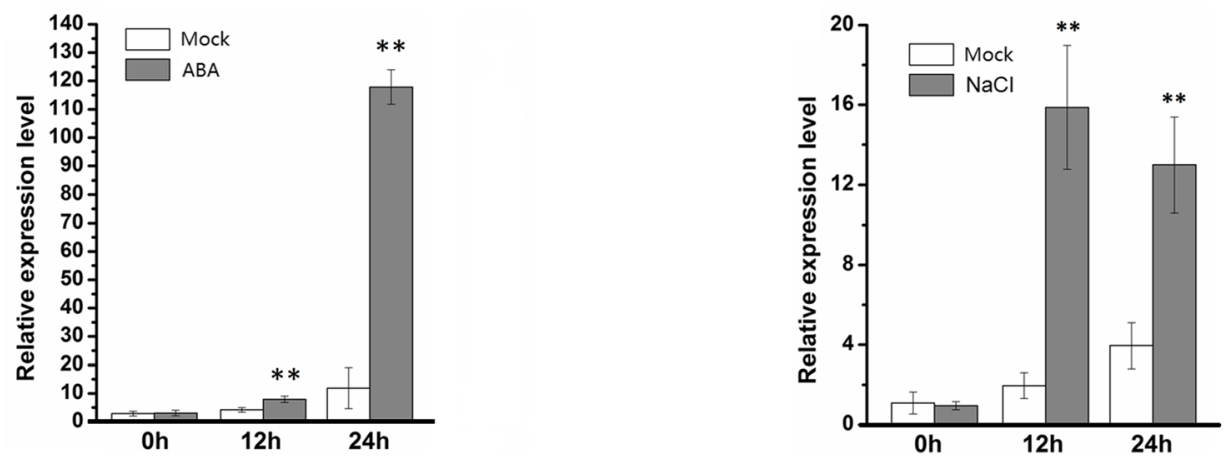

Fig. 1 Cloning and characterization of GhWIN2. (a) Phylogenetic analysis of WIN proteins from cotton (Gossypium hirsutum, Gh), rice (Oryza sativa, Os), barley (Hordeum vulgare, Hv), tomato (Solanum lycopersicum, SI), and Arabidopsis (At). Accession numbers: OsWR1(Os02g0202000); OsWR2(AK061 163); HvHUD (KP245804.1); AtWIN1/SHN1(AT1g15360); AtWIN2/SHN2(At5g11190); AtWIN3/SHN3(At5g25390); SISHN1(XP_004235965.1); SISHN2(XP_004251719.1); SISHN3(NM_001319202). The GhWIN2 is indicated by a red box. The rooted neighbor-joining tree was based on multiple sequence alignment using the MEGA5.1 software. (b) Subcellular localization of GhWIN2. GFP and DAPI fluorescence in the cotyledons of five-day-old plants of line 4 (scale bars: $10 \mu \mathrm{m})$. (C, D) The expression of GhWIN2 in two-week-old cotton seedlings after treatment with $100 \mu \mathrm{M} \mathrm{ABA}$ (c) and $100 \mathrm{mM} \mathrm{NaCl}(\mathbf{d})$. The mock samples were treated with double distilled water. Data were from three independent replicates and the results are means \pm SD. The relative transcription levels were normalized using GhUBQ7. Asterisks indicate significant differences between treated and mock plants according to Student's $t$-test $\left({ }^{*} P<0.05 ;{ }^{* *} P<0.01\right.$ )

\section{GhWIN2 activates the cuticle biosynthesis pathway}

To examine the functional similarity between GhWIN2 and other known WINs, we detected the expression level of several cuticle-related genes in the wild-type (WT) and transgenic Arabidopsis plants (lines 4 and 10). The selection of those genes was based on previous studies in which the WIN proteins that regulate genes were characterized $[17,38,39]$. The expression of the detected cutin biosynthesis-related genes AtGPAT6 (encoding glycerol-3-phosphate acyltransferase 6), AtGPDHc1 (encoding cytosolic G-3-P dehydrogenase), AtCYP86A4 (encoding cytochrome P450 enzymes), and AtCYP86A7 was greater in lines 4 and 10 than in the WT (Fig. 2a). Expression of the wax biosynthesis-related genes AtKCS1 (encoding 3-ketoacyl-CoA synthase 1), AtCER1 (encoding ECERIFERUM1), and AtCER2 (ECERIFERUM2) was greater in lines 4 and 10, whereas that of AtCER3 (ECERIFERUM3) was less, and that of AtCER6 (ECERIFERUM6) was unchanged, relative to expression in the WT. Expression of the cutin and wax biosynthesis-related gene AtLACS2 (encodes long-chain acyl-coenzyme A synthetase) was greater in lines 4 and 10 than in the WT.

We detected expression levels of the putative cotton orthologs of known Arabidopsis cuticle biosynthesis-related genes in GhWIN2 silenced in cotton plants (hereafter referred to as "TRV:GhWIN2 plants"; Additional file 1: Figure S4 and Figure S5). Expression of the detected cutin biosynthesis-related genes GhGPAT6, GhGPDHc1, GhCYP86A4, and GhCYP86A7 was lower in TRV:GhWIN2 plants than in the TRV:00. Expression of wax biosynthesis-related genes GhCER1 and GhCER6 were lower, whereas that of GhCER3 was unchanged, relative to expression in the TRV:00. Expression of 


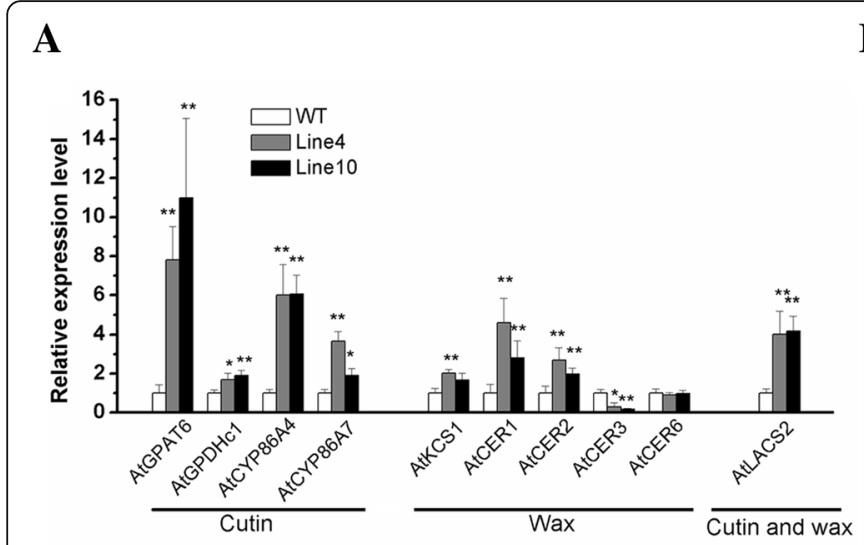

B

C
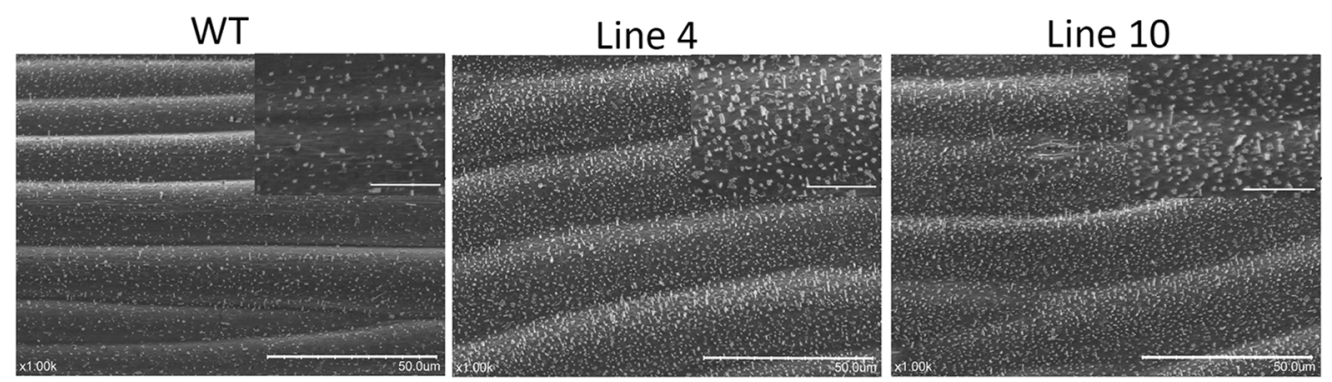

D

WT

Line 4
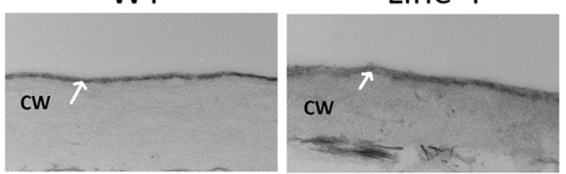

Line 10

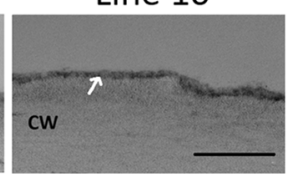

E

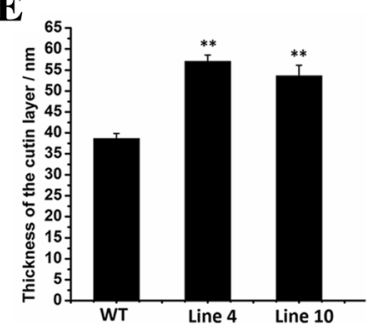

Fig. 2 GhWIN2 regulation of cuticle development. (a) Expression of genes involved in cuticle biosynthesis in four-week-old WT and transgenic Arabidopsis plants. Arabidopsis elongation factor-1-a gene (EF-1-a) was the endogenous reference for normalization. Data represent means \pm SD for three biological replicates. Student's $t$-test; ${ }^{*} P<0.05,{ }^{* *} P<0.01$. (b) Expression of the putative cuticle biosynthesis-related genes in cotton plants 14 days after infiltration. (c) SEM images of the stems of the WT and transgenic Arabidopsis plants. (Scale bars in main image, $50 \mu \mathrm{m}$; scale bars in insets, 10 um) (d) TEM images of the cuticle of the upper leaf surface (scale bar: $500 \mathrm{~nm}$ ). CW, cell wall; white arrows mark the cuticle. Images were taken at 50,000 $\times$ magnification. (e) The thickness of the cuticle layer from the leaf upper surface (d). Data are presented as means \pm SD from at least four independent biological replicates. Asterisks denote Student's $t$ test significance compared with WT plants $\left({ }^{*} P<0.05\right.$; $\left.{ }^{* *} P<0.01\right)$

GhLACS2 was also lower in TRV:GhWIN2 plants than in the TRV:00 plants (Fig. 2b). Overall, GhWIN2 up-regulated the expression of the most cuticle biosynthesis-related genes in plants.

Scanning electron microscopy revealed that the transgenic Arabidopsis plants had greater wax crystal accumulation in the stem and a thicker abaxial cuticle layer in the leaf than the WT plants (Fig. 2c, d, and e). These results indicate that GhWIN2 activates the cuticle biosynthesis pathway.

\section{GhCYP86A4 is the target gene of GhWIN2}

To explore the role of GhWIN2 in transcriptional activation, we cloned the promoters of GhLACS2, GhCYP86A4, and GhCYP86A7 genes. Using an in vivo transient gene expression assay, we found that only co-infiltration of $35 S$ : GhWIN2 and GhCYP86A4 $4_{\text {pro }}-L U C$ constructs resulted in transcriptional activation (Fig. 3a). Dehydration-responsive element-binding (DREB) and WIN transcriptional factors all belong to the subgroup of AP2/EREBP family that contains an AP2/EREBP domain involved in DNAbinding. Sequence alignment showed that GhWIN2 shares significant sequence identity with other AP2/EREBP proteins at the AP2/EREBP domain (Fig. 3b). From previous studies of DREB proteins, the single amino acid substitution of valine to alanine was sufficient to nullify the interaction between protein and DNA [40-42]. The corresponding residues are conserved in WIN proteins (Fig. 3b). Thus, we generated GhWIN2 $2^{V-A}$ with alanine substitution at the corresponding position. Co-infiltration 


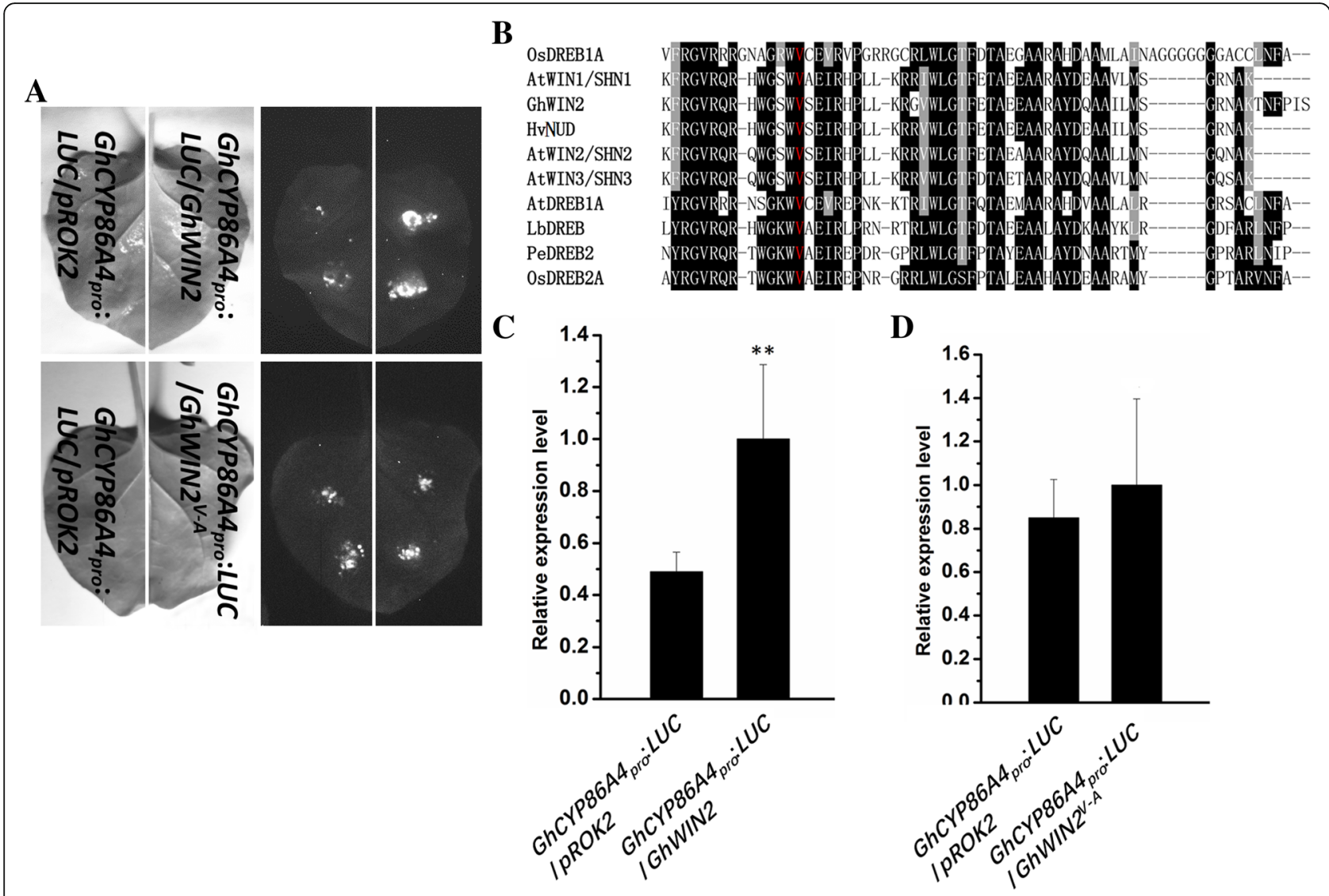

Fig. 3 GhWIN2 activation of the promoter of the GhCYP86A4 gene. (a) Luminescence signal of transient co-expression of GhWIN2/

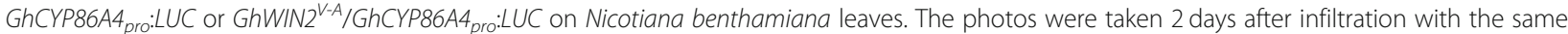
amount of Agrobacterium tumefaciens cultures containing the specified constructs. Two sites were injected for every treatment. (b) Sequence alignments of AP2/EREBP proteins. The conserved valine is marked in red. GenBank accession numbers: Oryza sativa OsDREB1A (AAN02486), OsDREB2A (AAN02487), AtDREB1A (Q9M0L0), Limonium bicolor LbDREB (ACP18975), Populus euphratica PeDREB2 (ABL86587), Hordeum vulgare HvNUD (AKF40403). (c, d) Expression of LUC transcripts 2 days after co-infiltration of Agrobacterium cells harboring GhWIN2/GhCYP86A4 pro:LUC (c) and GhWIN2 ${ }^{V-A}$ / GhCYP86A4 pro:LUC (d). Data represent means \pm SD from three independent biological replicates; Student's $t$-test; * $P<0.05,{ }^{* *} P<0.01$

of 35S:GhWIN2 $2^{V 19 A}$ and GhCYP86A4 pro-LUC constructs nullified the transcriptional activation (Fig. 3a). In addition, qPCR analysis showed that co-infiltration of 35S:GhWIN2 and GhCYP86A4 $4_{\text {pro }}-L U C$ activated the expression levels of the $L U C$ gene, while co-infiltration of 35S:GhWIN2 $2^{V 19 A}$ and GhCYP86A4 pro-LUC did not (Fig. 3c and d).

\section{ABA regulates the expression of cuticle biosynthesis genes mediated by GhWIN2}

We found that exogenous ABA strongly induced the expression of GhWIN2 (Fig. 1c). To further study the relationship between ABA and GhWIN2, we silenced GhPYL1 and GhNCED1, two key ABA biosynthesis genes in cotton [43, 44], and detected the expression levels of GhWIN2 in TRV:GhPYL1 and TRV:GhNCED1 plants (Additional file 1: Figure S6). Expression of GhWIN2 in TRV:GhPYL1 and TRV:GhNCED1 plants was suppressed after silencing of GhPYL1 and GhNCED1 (Fig. 4a).

To further identify the role of GhWIN2 in ABA-cuticle pathway, expression levels of GhCYP86A4, GhCYP86A7, and GhLACS2 was detected in TRV:00 and TRV: GhWIN2 plants. We found that exogenous ABA induced expression of these three genes in TRV:00 and TRV: GhWIN2 plants, whereas the inducible multiple was lower in TRV:GhWIN2 plants (Fig. 4b-d). These results suggest that ABA induces the expression of cuticle-related genes mediated by GhWIN2.

\section{GhWIN2 negatively regulates plant resistance to fungal pathogens}

Previous studies have shown that WIN orthologs positively or negatively regulate plant immune responses, depending on the plant species studied [19, 38]. Here, we were interested in finding out how GhWIN2 functions in cotton immune response. To examine this, we 

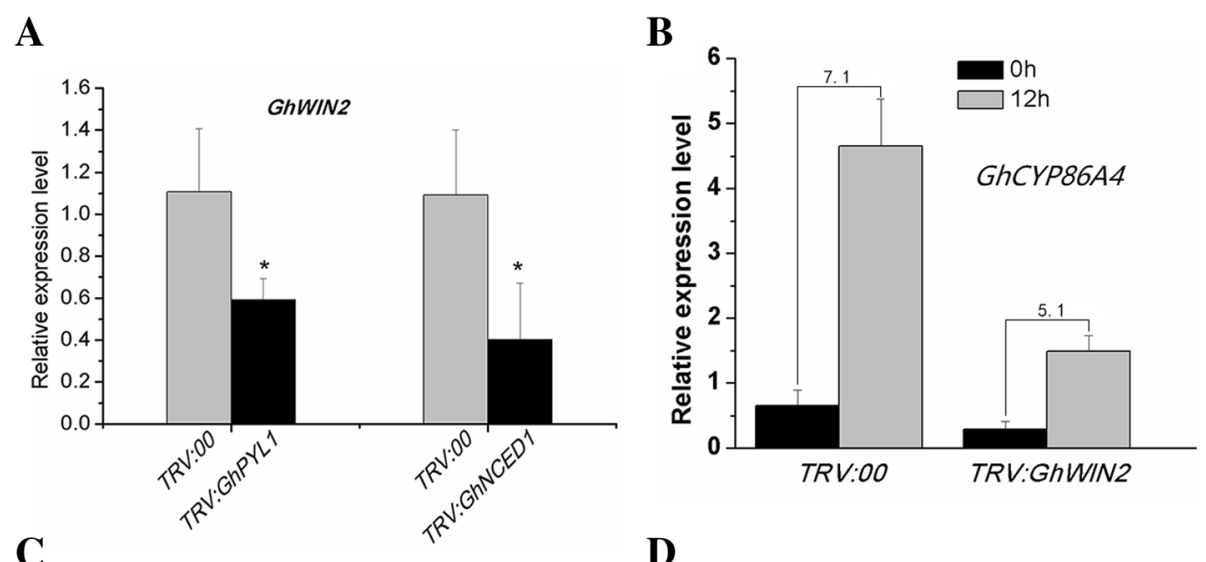

C

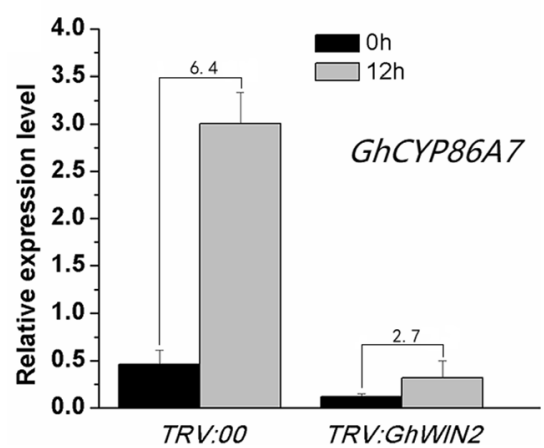

D

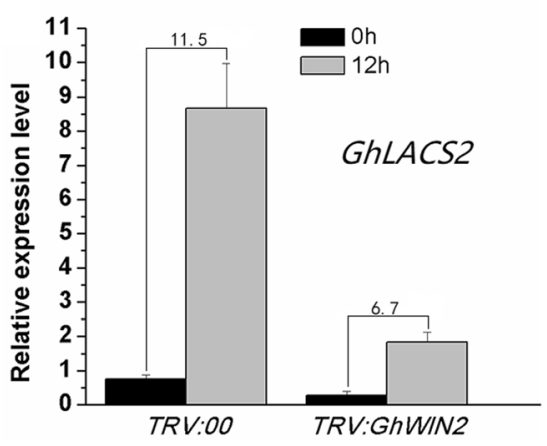

Fig. 4 GhWIN2 involvement in the ABA-cuticle pathway. (a) Expression levels of GhWIN2 were detected in TRV:GhPYL1 and TRV:GhNCED1 plants 14 days after infiltration. Values represent the means \pm SD from at least three independent biological replicates. Student's $t$-test; ${ }^{*} P<0.05$. (B-D) Expression levels of GhCYP86A4 (b), GhCYP86A7 (c), and GhLACS2 (d) in TRV:00 and TRV:GhWIN2 plants after treatment with $100 \mu M$ ABA. The numbers represent the inducible multiples of plants treated with ABA compared to untreated plants

challenged TRV:GhWIN2 and TRV:00 plants with $V$. dahliae. We detected greater resistance to $V$. dahliae in $T R V: G h W I N 2$ plants than in the TRV:00 plants (Fig. 5a). An analysis of relative fungal biomass showed that there was less fungus in TRV:GhWIN2 plants (Additional file 1: Figure S7). JA is involved in plant immune response to $V$. dahliae $[45,46]$. Surprisingly, expression of the putative or identified JA biosynthesis-related genes was suppressed and content of JA decreased in TRV: GhWIN2 plants (Fig. 5b and c). However, the content of SA and expression of the SA-responsive genes GhPR1 and GhPR5 was significantly greater in TRV:GhWIN2 plants than in TRV:00 plants (Fig. 5d and e). To determine whether SA enhanced the immune response, we decreased the content of SA by silencing the putative SA biosynthesis gene GhICS2 (TRV:GhICS2) [47]. Challenging with $V$. dahliae resulted in lower SA content in the TRV:GhICS2 plants and the TRV:GhWIN2/TRV:GhICS2 two-gene-silenced plants than in the TRV:00 and TRV: GhWIN2 plants (Additional file 1: Figure S8). Resistance to $V$. dahliae was lower in the two-gene-silenced plants than in the TRV:GhWIN2 plants (Fig. 5a; Additional file 1: Fig. S7). These results indicate that SA enhanced resistance to $V$. dahliae in TRV:GhWIN2 plants.
Next, we detected expression of genes involved in the fatty acid biosynthesis pathway upstream of the cuticle and JA biosynthesis pathway, and assessed the stearic acid content (Fig. 6). We found that expression levels of GhFATA, GhFATB, GhSAD3, GhSAD7, and GhKASII [48-51] were lower in TRV:GhWIN2 plants than in $T R V: 00$ plants. Content of the stearic acid was also lower in TRV:GhWIN2 plants than in TRV:00 plants.

\section{Discussion}

The aerial parts of land plants are covered by a cuticle, a hydrophobic layer that prevents the epidermal cells from having direct contact with the environment [7]. Many studies about the cuticle have been reported. However, the relationship between the cuticle biosynthesis pathway and other metabolic pathways has rarely been reported. To obtain deeper insight into the cuticle biosynthesis pathway, we cloned GhWIN2 from cotton. We have identified GhWIN2 as a positive regulator of the cuticle synthesis pathway. Our results provide evidence that reduced expression of GhWIN2 negatively regulates JA accumulation and positively regulates SA accumulation, and further confers resistance against $V$. dahliae. 


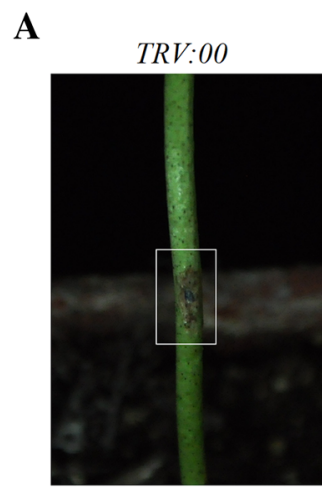

B

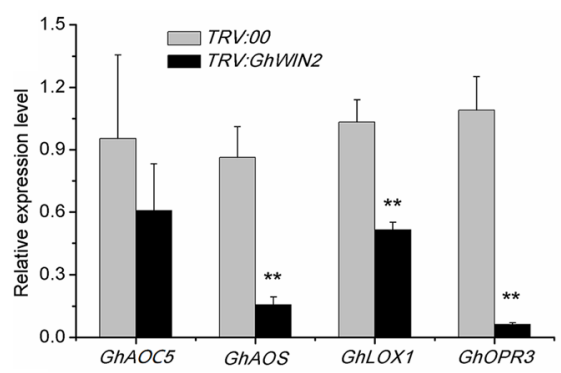

C

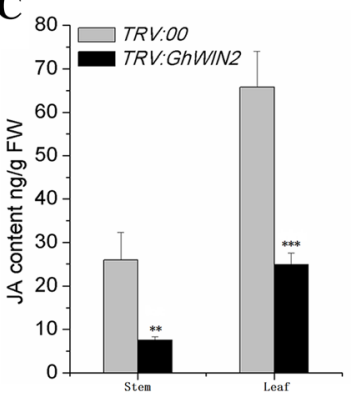

TRV:GhWIN2/

TRV:GhICS2

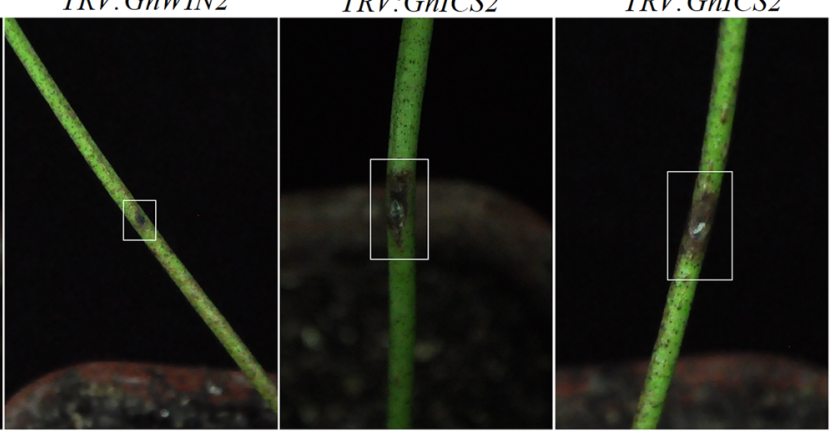

D

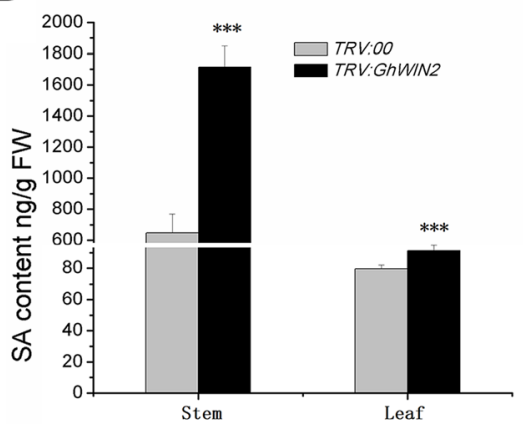

$\mathbf{E}$

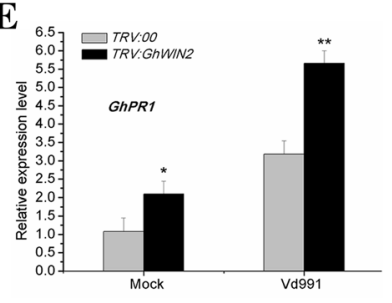

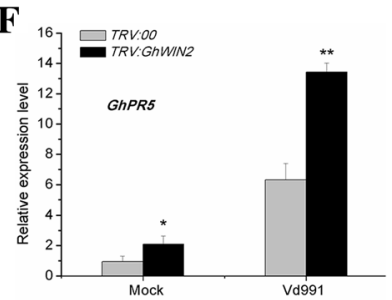

Fig. 5 GhWIN2 negative regulation of plant immune response to $V$. dahliae. (a) Disease symptoms after inoculation with $V$. dahliae. (b) Expression of JA biosynthesis-related genes in TRV:00 and TRV:GhWIN2 plants 14 days after agroinfiltration. Values are shown as means \pm SD from at least three independent biological replicates. Student's $t$-test; ${ }^{*} P<0.05$. (C, D) Content of JA (c) and SA (d). Values are the means \pm SD from six independent biological replicates. Student's $t$-test; ${ }^{*} P<0.05,{ }^{*} P<0.01,{ }^{* * *} P<0.001$. (e, $\mathbf{f}$ ) Expression of marker genes involved in SA response

It is known that WIN transcription factors transcriptionally activate the expression of cuticle synthesis-related genes $[13,52]$, resulting in cuticle accumulation. In barley, the Nud gene, which is homologous to the Arabidopsis WIN1/SHN1, is responsible for the control of a lipid biosynthesis pathway, generating organ adhesion [18]. We found that GhWIN2 belongs to the WIN protein family; first, it is localized at the nucleus (Fig. 1b); second, its overexpression in Arabidopsis increased the amount of wax crystals on the stem and thickness of abaxial cuticle in the leaf (Fig. 2c, d, and e); third, it transcriptionally regulated the expression of cuticle-related genes (Fig. 2a and b; Fig. 3).

Previous studies have shown that WIN orthologs transcriptionally activate target genes $[19,37,53]$. Here, to explore the role of GhWIN2 in transcriptional activation, we cloned the promoters of GhLACS2, GhCYP86A4, and GhCYP86A7 genes; we selected these genes based on previous studies in which WIN orthologs target genes were identified [19, 37, 53]. We found that GhWIN2 only activates the transcription of GhCYP86A4 promoter (Fig. 3a).

The DREB/ERF-type transcription factor belongs to the AP2/EREBP family [40]. DREB proteins contain two conserved amino acids in the AP2/EREBP domain, of which valine is especially important for DNA-binding [40-42]. Consistent with those findings, we found that GhWIN2 is a subfamily in the AP2/EREBP family, and contains a conserved AP2/EREBP domain (Fig. 3b). These analyses point to the potential role of GhWIN2 in 


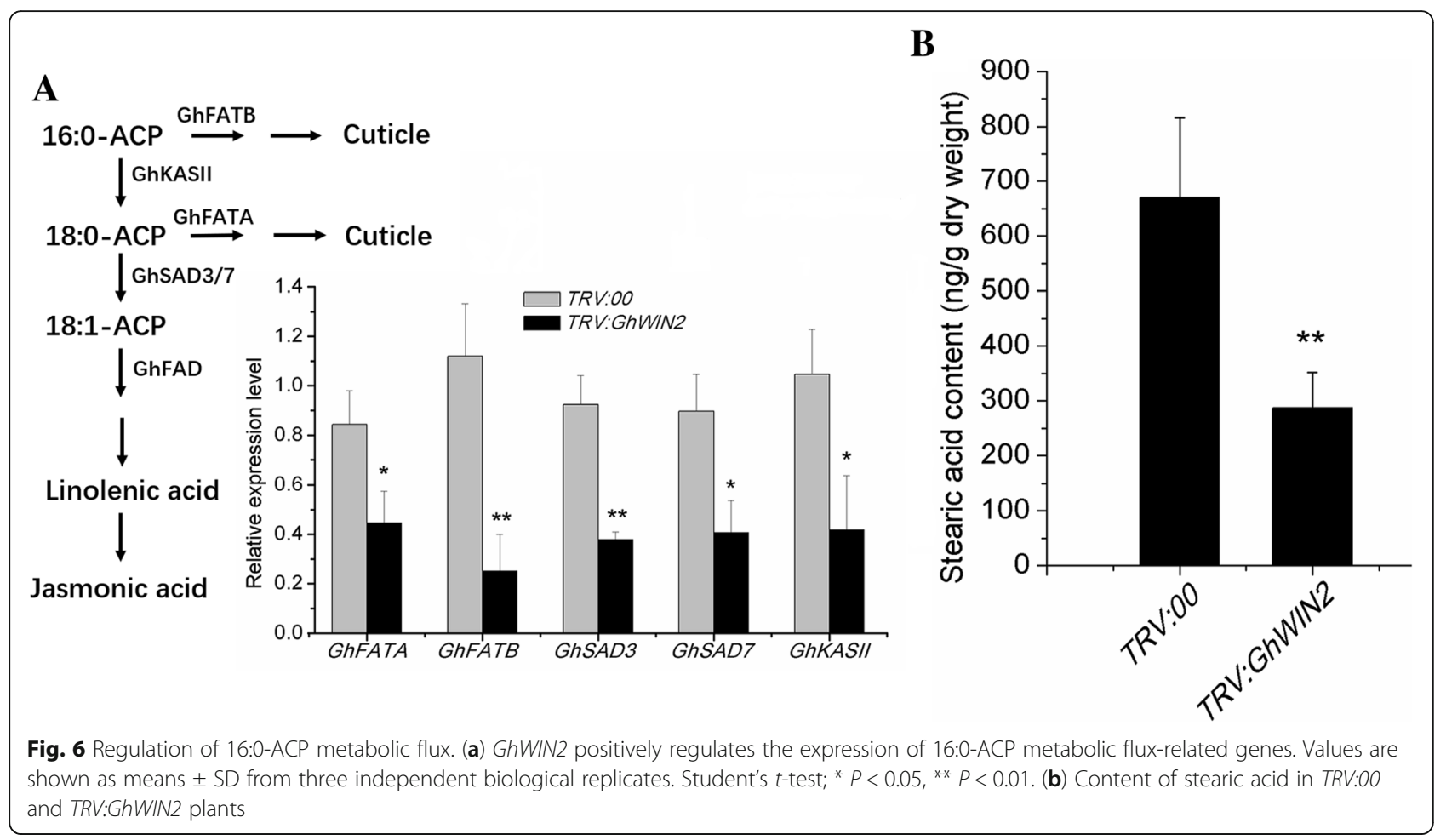

transcriptional activation of the corresponding conserved valine. We observed that the single amino acid substitution of valine to alanine was sufficient to nullify the transcriptional activation of GhWIN2 (Fig. 3a).

In Arabidopsis, it has been shown that the expression of many cuticle-related genes is suppressed or induced in ABA biosynthesis mutants, depending on the mutant used and the genes studied [54]. Exogenous ABA inhibits expression of cuticle biosynthesis-related genes in Physcomitrella patens, whereas those orthologs were induced in Arabidopsis [54]. These results led us to detect how ABA regulates expression of cuticle-related genes in cotton. Here, we found that exogenous ABA strongly induced expression of GhWIN2 (Fig. 1c). This is consistent with previous reports that TdWIN1 was strongly induced by ABA in wheat [55]. In addition, expression of GhWIN2 was suppressed in TRV:GhPYL1 and TRV: GhNCED1 plants (Fig. 4a). These results indicate that GhWIN2 is an ABA-responsive transcription factor. In Arabidopsis, the genes MYB16, MYB94, MYB96 and DE$W A X$ are involved in the ABA-cuticle regulatory pathway [54]. There have been no reports that AtWIN1/ SHN1 is involved in ABA-cuticle pathway. Here, we found that $\mathrm{ABA}$ induced some cuticle biosynthesis genes and that this was mediated, or partially mediated, by GhWIN2 (Fig. 4b-d). Thus, the mechanism of cuticle biosynthesis regulated by ABA is conserved and sophisticated in various species. As previously reported, although ABA generally induces expression of the transcription factors that positive regulate of cuticle biosynthesis, it also suppresses expression of one of these positive regulators, HDG1 [54].

Previous studies have shown that WIN orthologs positively or negatively regulate immune responses, depending on the plant and pathogen species studied. In Arabidopsis, overexpression of AtWIN1/SHN1 caused reduced expression of $P D F 1.2$, which compromised resistance to Botrytis cinerea and Alternaria brassicicola [56]. However, tomato SlWIN3/SHN3 conferred resistance in fruit against the fungus Colletotrichum coccodes, by causing a thickened cuticle that prevents fungal penetration [19]. Here, we found that GhWIN2 conferred sensitivity to $V$. dahliae (Fig. 5a). The thickened cuticle may contribute to the increased immune response, but its efficacy against $V$. dahliae was not great. Expression of PDF1.2 in AtWIN1/SHN1 overexpressed Arabidopsis plants was dramatically lower than in wild-type Arabidopsis plants [56]. In addition, the JA and cuticle biosynthesis pathways share the same precursor, stearic acid [57-60]. Therefore, reduced expression of GhWIN2 may lead to increased biosynthesis of JA, and this could explain why we the detected expression of JA biosynthesisrelated genes (Fig. 7). Expression of putative or identified JA biosynthesis-related genes and content of JA was lower in TRV:GhWIN2 plants than in TRV:00 plants (Fig. $5 \mathrm{~b}$ and $\mathrm{c}$ ), probably because that the precursor of JA biosynthesis reduced caused by decreased expression of GhWIN2. Thus, GhWIN2 may regulate the fatty acid 


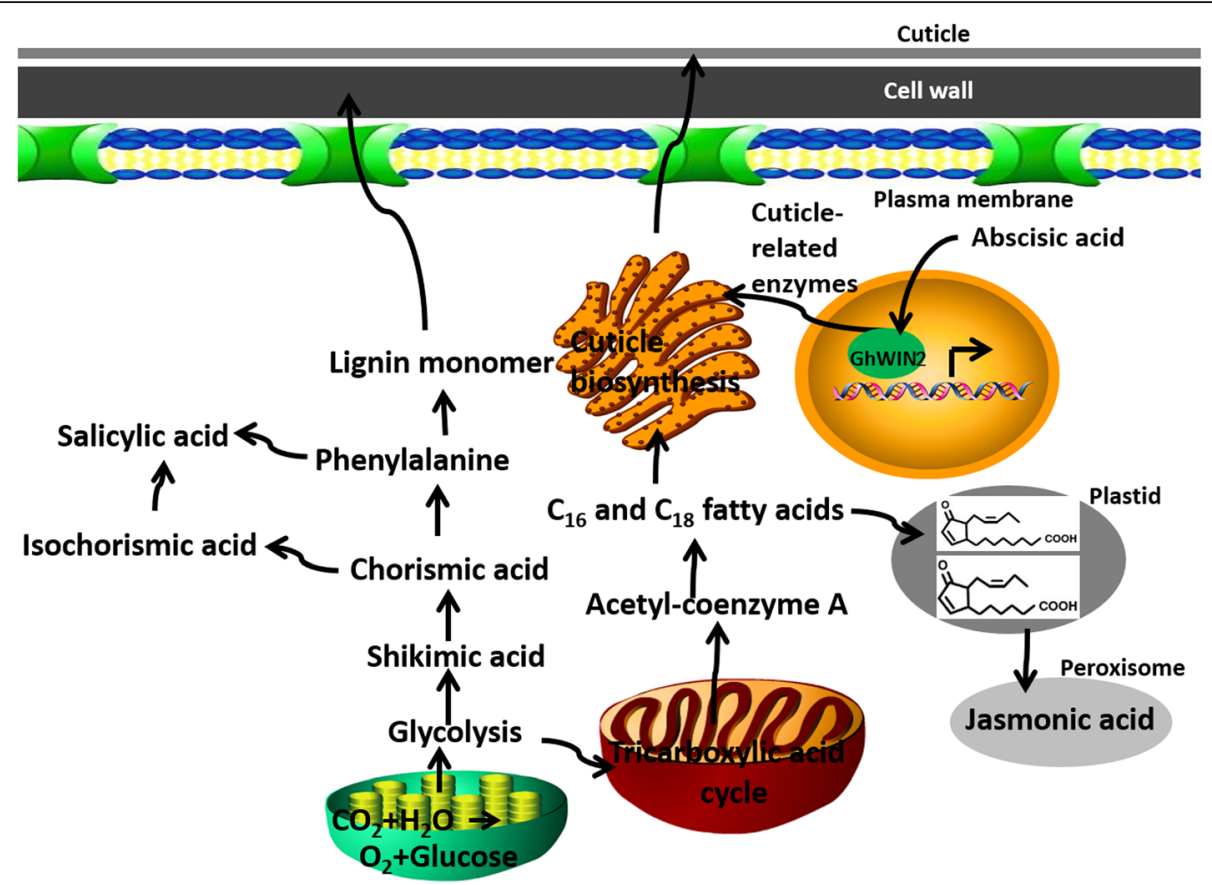

Fig. 7 Model of GhWIN2-related metabolic pathways. GhWIN2 positively regulated cuticle biosynthesis and content of JA, and negatively affected SA biosynthesis

biosynthesis pathway upstream of the cuticle biosynthesis pathway and the JA biosynthesis pathway simultaneously. As we predicted, the expression of genes related to stearic acid biosynthesis and stearic acid metabolism was reduced in TRV:GhWIN2 plants (Fig. 6). These results indicated that GhWIN2 regulated not only the cuticle biosynthesis pathway, but also the upstream pathways.

Content of SA and SA-responsive marker genes was substantially lower in TRV:GhWIN2 plants than in TRV: 00 plants (Fig. $5 \mathrm{~d}$ and e). This is consistent with the finding that overexpression of AtWIN1/SHN1 in rice caused a 45\% reduction in lignin content [20]. Biosynthesis of SA and lignin share part of the same pathway, and shikimic acid is a precursor to SA and lignin (Fig. 7) . Thus, it is likely that WIN regulates biosynthesis of SA and lignin via the same mechanism. This is probably because abnormal expression of GhWIN2 alters metabolic flux redirection. The greater resistance to $V$. dahliae that we observed in the TRV:GhWIN2 plants was probably caused by the higher SA content in these plants. To evaluate this, we subjected the putative gene GhICS2 involved in SA biosynthesis to functional analysis [47]. VIGS constructs for GhWIN2 and GhICS2 were used together to generate two-gene silenced plants, TRV: GhWIN2/TRV:GhICS2. Content of SA and resistance to $V$. dahliae was lower in TRV:GhWIN2/TRV:GhICS2 plants than in TRV:GhWIN2 plants. Thus, we conclude that SA conferred resistance to $V$. dahliae in TRV: GhWIN2 plants.
We obtained eleven sequences of WIN/SHN orthologs in the cotton genome. However, we only cloned GhWIN2 from cotton seedings and did not detect the expression of other sequences, indicating that other orthologs may not be expressed at the seedling stage, or it may be just that we have not detected them. If the latter, silencing of GhWIN2 could have silenced other orthologs simultaneously considering their high homology. Thus, it is worth noting that the observed phenotypes may be the results of silencing of WIN/SHN orthologs.

\section{Conclusions}

From the point of view of systems biology, there are intricate connections among different metabolic pathways: they compete for substrates, have different metabolic kinetics, and their products can activate or inhibit other pathways [35]. Studying the connections between different metabolic pathways in plants is important in bioenergy and synthetic biology, and can expand our understanding of whole plant systems. The cuticle is related to plant drought tolerance and disease resistance. It acts as a barrier limiting non-stomatal water loss [4, 61, 62]. Few studies have examined the relationship between the cuticle biosynthesis pathway and other metabolic pathways. Here, we have provided evidence that GhWIN2 not only regulates cuticle biosynthesis pathway, but also positively influences JA biosynthesis and negatively influences SA biosynthesis. The other WIN/SHN 
orthologs may also be involved in regulation of these physiological processes.

\section{Methods}

\section{Plant and fungal cultivation}

The state cotton variety 2,006,001 (original strain no. GK44) was kindly provided by the Cotton Research Institute, Chinese Academy of Agricultural Sciences. Germination was accelerated before sowing in soil. The cotton seedlings were grown for 2 weeks at day/night temperatures of $26^{\circ} \mathrm{C} / 23^{\circ} \mathrm{C}$ in an incubator, using a $16 \mathrm{~h}$ light $/ 8 \mathrm{~h}$ dark photoperiod cycle.

Arabidopsis thaliana Col-0 wild type (WT) and transgenic Arabidopsis plants were grown in soil in an incubator at $23^{\circ} \mathrm{C}, 70 \%$ relative humidity, with a $16 \mathrm{~h}$ light / $8 \mathrm{~h}$ dark photoperiod. Seedlings were grown on agar plates containing $1 \%$ sucrose and $0.8 \%$ agar. Seeds were sterilized before being planted on the plate.

Verticillium dahliae strain Vd991 was cultured on a potato dextrose agar plate for 7 days at $26^{\circ} \mathrm{C}$ and then inoculated into Czapek medium for 1 week. The spore suspension $\left(10^{6}\right.$ spores $\left.\mathrm{ml}^{-1}\right)$ was then prepared by filtration.

\section{RNA extraction and RT-qPCR}

Total RNA was extracted from the transgenic Arabidopsis plants or treated cotton plants using a plant RNA extraction kit (Biomed). Two micrograms of total RNA were reverse transcribed using a Fast Quant cDNA Reverse Kit (Tiangen Biotech Co., Ltd., Beijing, China). RT-qPCR was carried out using a SYBR ${ }^{\bullet}$ Premix Ex Taq (Tli RNaseH Plus) (Takara, Shiga, Japan). The endogenous genes GhUBQ7 (DQ116441) and EF-1- $\alpha$ were used as the control in cotton and Arabidopsis plants, respectively. Reactions were amplified on an ABI7500 thermocycler (Applied Biosystems, Foster City, CA, USA). The transcription levels of GhWIN2 were analyzed by the comparative CT $\left(2^{-\triangle \Delta C T}\right)$ method. Relative fungal biomass was detected by RT-qPCR. The $V$. dahliae specific primers, ITS1-F/ST-Ve1-R, were used (Additional file 1: Table S1).

\section{Subcellular localization}

To obtain GhWIN2-overexpressing Arabidopsis plants, GhWIN2 was amplified using the primers WIN-1300F/WIN-1300-R (Additional file 1: Table S1), with Pst1 and Spe1 cleavage sites at the $5^{\prime}$ and $3^{\prime}$ ends, respectively. Next, the sequence was inserted into a modified Super-pCAMBIA1300 vector (Additional file 1: Figure S2). The recombinant construct was transformed into Agrobacterium tumefaciens strain GV3101. GV3101 was then used to infect Arabidopsis thaliana to obtain transgenic plants [63].
Seedlings were grown for five days under standard conditions. To stain nuclei, five day-old transgenic seedlings were submerged in PBS containing $5 \mu \mathrm{g} / \mathrm{mL} 4^{\prime}-6$ diamidino-2-phenylindole (DAPI) (Sigma) and then incubated for 15 min before imaging [64]. Imaging of GFP and DAPI was performed with a FLUOVIEW FV1000 confocal laser scanning microscope (Olympus, Tokyo, Japan). GFP and DAPI were excited at $405 \mathrm{~nm}$ and 488 $\mathrm{nm}$, respectively.

\section{Transient gene expression assay}

The promoter sequence of GhCYP86A4 was cloned using primers GhCYP86A4 pro $-\mathrm{F} / \mathrm{GhCYP86A} 4_{\text {pro }}-\mathrm{R}$ (Additional file 1: Table S1). The Gateway cloning system (Invitrogen) was used. The promoter sequence was cloned into vector pGWB435, which contains an LUC reporter gene [65]. The construct GhCYP86A4 pro:LUC was then transformed into Agrobacterium tumefaciens strain GV3101. The GV3101 was cultured in LB medium containing $50 \mathrm{mg} /$ $\mathrm{mL}$ spectinomycin and $50 \mathrm{mg} / \mathrm{mL}$ rifampicin. The coding sequence of GhWIN2 and GhWIN2 $2^{\mathrm{V}-\mathrm{A}}$ was cloned into the vector pROK2 to generate 35S:GhWIN2 and 35S: GhWIN2 $2^{\mathrm{V}-\mathrm{A}}$. These two constructs were then transformed into Agrobacterium tumefaciens strain GV3101. GV3101 harboring 35S:GhWIN2 or 35S:GhWIN2 ${ }^{\mathrm{V}-\mathrm{A}}$ was then cultured in LB medium containing $50 \mathrm{mg} / \mathrm{mL}$ kanamycin and $50 \mathrm{mg} / \mathrm{mL}$ rifampicin. For transient expression assay in tobacco, the Agrobacterium tumefaciens strain GV3101 cells containing GhCYP86A4 pro:LUC and 35S:GhWIN2/35S: GhWIN2 ${ }^{\mathrm{V}-\mathrm{A}}$ were mixed and treated with infiltration buffer (200 mM acetosyringone, $10 \mathrm{mM}$ MES, pH 5.6; $10 \mathrm{mM}$ $\mathrm{MgCl}_{2}$ ) for $3 \mathrm{~h}$. Next, the mixed cultures were injected into the leaf using a needleless syringe. The plants were then grown in the dark for $24 \mathrm{~h}$ and then under normal growth conditions for $48 \mathrm{~h}$. The LUC signal was measured using a CCD camera (1300B; Roper) after being sprayed with $1 \mathrm{mM}$ luciferin (Sigma-Aldrich).

\section{Virus-induced gene silencing}

The fragments of GhWIN2 was amplified and cloned into an improved $p T R V 2$ virus-induced gene silencing (VIGS) vector, pYL192 [65-67]. The recombinant plasmid pTRV2:GhWIN2 was then transformed into Agrobacterium tumefaciens strain GV3101. Gene silencing was conducted according to the method described in $\mathrm{Li}$ et al. [65]. Agrobacterium cultures harboring $p T R V 1$, $p T R V 2$, or $p T R V 2: G h W I N 2$ were grown in LB medium containing $50 \mu \mathrm{g} / \mathrm{mL}$ rifampicin, $50 \mu \mathrm{g} / \mathrm{mL}$ kanamycin, $20 \mu \mathrm{M}$ acetosyringone, and $10 \mathrm{mM}$ MES until the $\mathrm{OD}_{600}$ reached a value of 1 . The cotyledons of 14-day-old cotton seedlings were injected with a mixture of the Agrobacterium cultures harboring the $p T R V 1 / p T R V 2$ plasmids (1:1 ratio, v/v) as the control and $p T R V 1 /$ pTRV2:GhWIN2 plasmids (1:1 ratio, v/v) as the 
experimental group. We also constructed $p T R V 2$ : GhCLA1 (Cloroplastos alterados 1; $500 \mathrm{bp)}$ to detect the efficiency of silencing under our experimental conditions.

\section{Accession numbers}

AtGPAT6, AT2G38110; AtGPDHc1, AT2G41540; AtCY P86A4, AT1G01600; AtCYP86A7, AT1G63710; AtKCS1, AT1G01120; AtCER1, At1G02205; AtCER2, At4G24510; AtCER3, At5g57800; AtCER6, At1g68530; AtLACS2, At1G49430; GhGPAT6, ADK23938.1; GhGPDHc1, XP 016671566.1; GhCYP86A4, XM_016840837.1; GhCYP 86A7, XP_016719401.1; GhCER1, XP_016681695.1; GhC ER3, XP_016725538.1; GhCER6, KT625616.1; GhLA CS2, XP 016707966.1; GhAOC5, KF383427.1; GhAOS, ALG62633.1; GhLOX1, AF361893.4; GhOPR3, NP_0013 13917.1; GhFATA, XP_016727762.1; GhFATB, XP_0167 20478.1; GhSAD3, XM_016885870.1; GhSAD7, XM_ 016843547.1; GhKASII, HM236494.1; GhPYL1, XM 016815548.1; GhNCED1, HM014161.

\section{Additional file}

Additional file 1: Supplementary information. Figure S1 Amino acid sequence alignment of cotton WIN orthologs. Figure S2 Schematic representation of the plant expression vector pCAMBIA1300-GhWIN2. Figure S3 Relative expression of GhWIN2 in transgenic Arabidopsis plants. Figure S4 Expression of GhWIN2 in control and silenced cottons. Figure S5 VIGS-mediated silencing of GhCLA1 in cotton. Figure S6 Relative expression of GhPYL1 and GhNCED1 in cotton plants 14 days after agroinfiltration. Figure S7 Relative biomass of $V$. dahliae in infected cotton plants. Figure S8 VIGS-mediated silencing of GhICS2 in cotton. Table S1 Primers used in this study. (PDF $1037 \mathrm{~kb}$ )

\section{Abbreviations}

13-HPOT: 13-hydroperoxyoctadecatrienoic acid; ABA: Abscisic acid; C16/C18acyl-CoA: 16/18-carbon long-chain acyl-coenzyme A; CER1: ECERIFERUM1; CER2: ECERIFERUM2; CER3: ECERIFERUM3; CER6: ECERIFERUM6; CYP: Cytochrome P450 enzymes; DREB: Dehydration-responsive elementbinding; ECR: Enoyl-CoA reductase; FAEs: Fatty acid elongases; GPAT6: Glycerol-3-phosphate acyltransferase 6; GPDHc1: Cytosolic G-3-P dehydrogenase; HCD: 3-hydroxyacyl-CoA dehydratase; JA: jasmonic acid; KCR: $\beta$-ketoacyl-CoA reductase; KCS: $\beta$-ketoacyl-CoA synthase; KCS1: 3ketoacyl-CoA synthase 1; LACS2: Long-chain acyl-coenzyme A synthetase; LACSs: Long-chain acyl-CoA synthetases; LOX: lipoxygenase; $\mathrm{NaCl}$ : Sodium chloride; SA: Salicylic acid; VIGS: Virus-induced gene silencing; VLCFA: Verylong-chain fatty acid

\section{Acknowledgements}

We thank Prof. Yule Liu (Tsinghua University) for the VIGS vectors and Prof. Jinsong Zhang (Institute of Genetics and Developmental Biology, Chinese Academy of Sciences) for the vector pGWB435.

\section{Authors' contributions}

$Y H, F L$, and $X L$ designed experiments. $X L$ executed experiments. $X L, N L, Y S$, $\mathrm{DL}, \mathrm{PW}, \mathrm{YP}, \mathrm{XM}$, and $\mathrm{XG}$ analyzed data. $\mathrm{XL}$ wrote the manuscript. $\mathrm{YH}$ and $\mathrm{FL}$ supervised the project. All authors have read and approved the final version of the manuscript.

\section{Funding}

This work was sponsored by the 'Seven Crop Breeding' National Major Project (grant no. 2016YFD0101006); the National key research and development program "chemical fertilizers and pesticide reduction efficiency integrated technology research and development" (grant no. 2017YFD0201900); the State Key Laboratory of Cotton Biology (grant no. CB2019B04). These funding bodies had no influence on the design of the study, collection, analysis, interpretation of data, or the manuscript writing.

Availability of data and materials

The data sets during the current study are available from the corresponding author.

Ethics approval and consent to participate

Not applicable.

\section{Consent for publication}

Not applicable.

\section{Competing interests}

The authors declare that they have no competing interests.

Received: 31 January 2019 Accepted: 14 June 2019

Published online: 28 August 2019

\section{References}

1. Freimoser FM, Hu G, St Leger RJ. Variation in gene expression patterns as the insect pathogen Metarhizium anisopliae adapts to different host cuticles or nutrient deprivation in vitro. Microbiology. 2005;151(Pt 2:361

2. Ali S, Huang $Z$, Ren $S$. The role of diamondback moth cuticle surface compounds in pre-penetration growth of the entomopathogen Isaria fumosoroseus. J Basic Microb. 2010:50(5):411.

3. Bunn R. Histone $\mathrm{H} 2 \mathrm{~B}$ monoubiquitination is involved in the regulation of cutin and wax composition in Arabidopsis thaliana. Plant Cell Physiol. 2014; 55(2):455-66.

4. Pollard M, Beisson F, Li Y, Ohlrogge JB. Building lipid barriers: biosynthesis of cutin and suberin. Trends Plant Sci. 2008;13(5):236.

5. Samuels L, Kunst L, Jetter R. Sealing plant surfaces: cuticular wax formation by epidermal cells. Annu Rev Plant Biol. 2008:59.

6. Lü SY, Song T, Kosma DK, Parsons EP, Rowland O, Jenks MA. Arabidopsis CER8 encodes LONG-CHAIN ACYL-COA SYNTHETASE 1 (LACS1) that has overlapping functions with LACS2 in plant wax and cutin synthesis. Plant J. 2009;59(4):553-64

7. Yeats TH, Rose JKC. The formation and function of plant cuticles. Plant Physiol. 2013;163(1):5-20.

8. Han G, Gable K, Kohlwein SD, Beaudoin F, Napier JA, Dunn TM. The Saccharomyces cerevisiae YBR159w gene encodes the 3-ketoreductase of the microsomal fatty acid elongase. J Biol Chem. 2002:277(38):35440-9.

9. Beaudoin F, Gable K, Sayanova O, Dunn T, Napier JA. A Saccharomyces cerevisiae gene required for heterologous fatty acid elongase activity encodes a microsomal beta-keto-reductase. J Biol Chem. 2002;277(13): 11481-8.

10. Kohlwein SD, Eder S, Oh CS, Martin CE, Gable K, Bacikova D, et al. TsC13p is required for fatty acid elongation and localizes to a novel structure at the nuclear-vacuolar Interface in Saccharomyces cerevisiae. Mol Cell Biol. 2001; 21(1):109.

11. Hegebarth D, Buschhaus C, Joubã SJ, Thoraval D, Bird D, Jetter R. Arabidopsis ketoacyl-CoA synthase 16 forms C36 /C38 acyl precursors for leaf trichome and pavement surface wax. Plant Cell Environ. 2017:40(7).

12. Paul S, Gable K, Beaudoin F, Cahoon E, Jaworski J, Napier JA, et al. Members of the Arabidopsis FAE1-like 3-Ketoacyl-CoA synthase gene family substitute for the Elop proteins of Saccharomyces cerevisiae. J Biol Chem. 2006; 281(14):9018-29.

13. Aharoni A, Dixit S, Jetter R, Thoenes E, Van AG, Pereira A. The SHINE clade of AP2 domain transcription factors activates wax biosynthesis, alters cuticle properties, and confers drought tolerance when overexpressed in Arabidopsis. Plant Cell. 2004;16(9):2463-80.

14. Bernard A, Domergue F, Pascal S, Jetter R, Renne C, Faure JD, et al. Reconstitution of plant alkane biosynthesis in yeast demonstrates that Arabidopsis ECERIFERUM1 and ECERIFERUM3 are core components of a very-long-chain alkane synthesis complex. Plant Cell. 2012;24(7):3106-18.

15. Holmes MG, Keiller DR. A novel phototropic response to supplementary ultraviolet (UV-B and UV-A) radiation in the siliquas of oilseed rape (Brassica napus L.) grown under natural conditions. Photoch Photobio Sci. 2002;1(11): $890-5$. 
16. Li F, Wu X, Lam P, Bird D, Zheng H, Samuels L, et al. Identification of the wax ester synthase/acyl-coenzyme a: diacylglycerol acyltransferase WSD1 required for stem wax ester biosynthesis in Arabidopsis. Plant Physiol. 2008; 148(1):97-107.

17. Broun $P$, Poindexter $P$, Osborne E, Jiang CZ, Riechmann JL. WIN1, a transcriptional activator of epidermal wax accumulation in Arabidopsis. Proc Natl Acad Sci U S A. 2004;101(13):4706-11.

18. Taketa S, Amano S, Tsujino Y, Sato T, Saisho D, Kakeda K, et al. Barley grain with adhering hulls is controlled by an ERF family transcription factor gene regulating a lipid biosynthesis pathway. Proc Natl Acad Sci U S A. 2008; 105(10):4062-7.

19. Shi JX, Adato A, Alkan N, He Y, Lashbrooke J, Matas AJ, et al. The tomato SISHINE3 transcription factor regulates fruit cuticle formation and epidermal patterning. New Phytol. 2013;197(2):468-80.

20. Ambavaram MM, Krishnan A, Trijatmiko KR, Pereira A. Coordinated activation of cellulose and repression of lignin biosynthesis pathways in rice. Plant Physiol. 2011;155(2):916-31.

21. Parchmann S, Gundlach H, Mueller MJ. Induction of 12-Oxo-Phytodienoic acid in wounded plants and elicited plant cell cultures. Plant Physiol. 1997; 115(3):1057-64.

22. Grechkin AN, Fazliev FN, Mukhtarova LS. The lipoxygenase pathway in garlic ( Allium sativum L.) bulbs: detection of the novel divinyl ether oxylipins. FEBS Lett. 1995;371(2):159.

23. Ando A, Ogawa J, Kishino S, Ito T, Shirasaka N, Sakuradani E, et al. Fatty acid desaturation and elongation reactions of Trichoderma sp. 1-OH-2-3. Journal of the American Oil Chemists Society. 2009;86(3):227-33.

24. Kolattukudy PE. Biosynthesis and degradation of lipid Polymers 1977.

25. Yalpani N, Silverman P, Wilson TM, Kleier DA, Raskin I. Salicylic acid is a systemic signal and an inducer of pathogenesis-related proteins in virusinfected tobacco. Plant Cell. 1991;3(8):809-18.

26. Derksen $H$, Badawi M, Henriquez MA, Zhen Y, El-Bebany AF, Daayf F. Differential expression of potato Defence genes associated with the salicylic acid Defence Signalling pathway in response to weakly and highly aggressive isolates of Verticillium dahliae. J Phytopathol. 2013;161(3):142-53.

27. Mo HJ, Sun YX, Zhu XL, Wang XF, Zhang Y, Yang J, et al. Cotton Sadenosylmethionine decarboxylase-mediated spermine biosynthesis is required for salicylic acid- and leucine-correlated signaling in the defense response to Verticillium dahliae. Planta. 2016;243(4):1023-39.

28. Mo H, Wang X, Zhang Y, Zhang G, Zhang J, Ma Z. Cotton polyamine oxidase is required for spermine and camalexin signalling in the defence response to Verticillium dahliae. Plant J. 2015;83(6):962-75.

29. Xu L, Zhang W, He X, Liu M, Zhang K, Shaban M, et al. Functional characterization of cotton genes responsive to Verticillium dahliae through bioinformatics and reverse genetics strategies. J Exp Bot. 2014;65(22):667992.

30. Dempsey DA, Vlot AC, Wildermuth MC, Klessig DF. Salicylic acid biosynthesis and metabolism. Arabidopsis Book. 2011:9:e0156.

31. Brown SA, Neish AC. Shikimic acid as a precursor in lignin biosynthesis. Nature. 1955:175(4459):688-9.

32. Pascal $R$, Juliette $P$, Delphine $G$, Laure D, Michel M. Tyrosine and phenylalanine are synthesized within the plastids in Arabidopsis. Plant Physiol. 2009;149(3):1251-60.

33. Boerjan W, Ralph J, Baucher M. LIGNIN BIOSYNTHESIS. Annu Rev Plant Biol. 2003;54(1):519-46.

34. Ideker T, Galitski TL. A new approach to decoding life: systems biology. Annu Rev Genomics Hum Genet. 2001;2(1):343-72.

35. Ideker $T$, Winslow LR, Lauffenburger AD. Bioengineering and systems biology. Ann Biomed Eng. 2006;34(7):1226-33.

36. Robertseilaniantz A, Grant M, Jones JDG. Hormone crosstalk in plant disease and defense: more than just jasmonate-salicylate antagonism. Annu Rev Phytopathol. 2011;49(1):317-43

37. Kannangara R, Branigan C, Liu Y, Penfield T, Rao V, Mouille G, et al. The transcription factor WIN1/SHN1 regulates Cutin biosynthesis in Arabidopsis thaliana. Plant Cell. 2007;19(4):1278-94.

38. Sela D, Buxdorf K, Shi JX, Feldmesser E, Schreiber L, Aharoni A, et al. Overexpression of AtSHN1/WIN1 provokes unique defense responses. PLoS One. 2013;8(7):e70146.

39. Kong $L$, Chang C. Suppression of wheat TaCDK8/TaWIN1 interaction negatively affects germination of Blumeria graminis f.sp. tritici by interfering with very-long-chain aldehyde biosynthesis. Plant Mol Biol. 2018;96(1-2):1-14
40. Sakuma Y, Liu Q, Dubouzet JG, Abe H, Shinozaki K, Yamaguchi-Shinozaki K. DNA-binding specificity of the ERF/AP2 domain of Arabidopsis DREBs, transcription factors involved in dehydration- and cold-inducible gene expression. Biochemical \& Biophysical Research Communications. 2002; 290(3):998-1009.

41. Hong JP, Kim WT. Isolation and functional characterization of the caDREBLP1 gene encoding a dehydration-responsive element binding-factorlike protein 1 in hot pepper ( Capsicum annuum L. cv. Pukang). Planta. 2005;220(6):875-88.

42. Gao S, Xu H, Cheng X, Ming C, Xu Z, Li L, et al. Improvement of wheat drought and salt tolerance by expression of a stress-inducible transcription factor gm DREB of soybean ( Glycine max ). Chin Sci Bull. 2005;50(23):271423.

43. Liang C, Liu Y, Li Y, Meng Z, Yan R, Zhu T, et al. Activation of ABA receptors gene GhPYL9-11A is positively correlated with cotton drought tolerance in transgenic Arabidopsis. Front Plant Sci. 2017;8:1453.

44. Zhang G, Lu T, Miao W, Sun L, Tian M, Wang J, et al. Genome-wide identification of ABA receptor PYL family and expression analysis ofPYLsin response to $A B A$ and osmotic stress inGossypium. Peerj. Web Server issue. 2017;5:e4126.

45. Gao W, Long L, Zhu LF, Xu L, Gao WH, Sun LQ, et al. Proteomic and virusinduced gene silencing (VIGS) analyses reveal that gossypol, brassinosteroids, and jasmonic acid contribute to the resistance of cotton to Verticillium dahliae. Mol Cell Proteomics. 2013;12(12):3690-703.

46. Li X, Pei Y, Sun Y, Liu N, Wang P, Liu D, et al. A cotton Cyclin-dependent kinase $\mathrm{E}$ confers resistance toVerticillium dahliaeMediated by Jasmonateresponsive pathway. Front Plant Sci. 2018;9:642.

47. Cindy F, Mario S, Eliane AM, Jean-Pierre M, Floriane LH. Salicylic acid and its location in response to biotic and abiotic stress. FEBS Lett. 2011;585(12): 1847-52.

48. Litao $Y$, Jianxiu C, Cheng H, Yuhui L, Shirong J, Liangwen P, et al. Validation of a cotton-specific gene, Sad1, used as an endogenous reference gene in qualitative and real-time quantitative PCR detection of transgenic cottons. Plant Cell Rep. 2005:24(4):237-45

49. Carlsson AS, Labrie ST, Kinney AJ, Penny WK, John B. A KAS2 cDNA complements the phenotypes of the Arabidopsis fab1 mutant that differs in a single residue bordering the substrate binding pocket. Plant J. 2010;29(6): 761-70.

50. Dong J, Wei LB, Hu Y, Zhang TZ, Guo WZ. Molecular cloning and expression analysis of four novel fatty acid synthesis related genes in Gossypium hirsutum L. Acta Agron Sin. 2010;(12):2084-90.

51. Liu RZ, Liu Q, Green A. Cotton transformation based on inverted repeat construct of oleic acid desaturase gene GhFAD 2-1. Shandong Agricultural Sciences. 2001;6(6):18-22.

52. Samuels AL, Jetter R, Kunst L, Pollard M, Ohlrogge J. Cuticular lipid composition, surface structure, and gene expression in Arabidopsis stem epidermis. Plant Physiol. 2005;139(4):1649-65.

53. Jian Xin S, Sergey M, Sheron DO, Caroline B, Franke RB, Lukas S, et al. SHINE transcription factors act redundantly to pattern the archetypal surface of Arabidopsis flower organs. PLoS Genet. 2011;7(5):e1001388.

54. Cui F, Brosché $M$, Lehtonen $M$, Amiryousefi $A$, Xu E, Punkkinen $M$, et al. Dissecting abscisic acid signaling pathways involved in cuticle formation. Mol Plant. 2016:9(6):926-38.

55. Djemal R, Khoudi H. Isolation and molecular characterization of a novel WIN1/SHN1 ethylene-responsive transcription factor TdSHN1 from durum wheat ( Triticum turgidum . L. Subsp. durum ). Protoplasma. 2015;252(6): 1461-73.

56. Zhu Y, Schluttenhoffer CM, Wang P, Fu F, Thimmapuram J, Zhu JK, et al CYCLIN-DEPENDENT KINASE8 differentially regulates plant immunity to fungal pathogens through KINASE-dependent and -independent functions in Arabidopsis. Plant Cell. 2014;26(10):4149-70

57. Marmey P, Jalloul A, Alhamdia M, Assigbetse K, Cacas JL, Voloudakis AE, et al. The 9-lipoxygenase GhLOX1 gene is associated with the hypersensitive reaction of cotton Gossypium hirsutum to Xanthomonas campestris pv malvacearum. Plant Physiology \& Biochemistry. 2007;45(8):596-606.

58. Yuange W, Huaihua, Qingquo. Improvement of copper tolerance of Arabidopsis by transgenic expression of an allene oxide cyclase gene, GhA OC1, in upland cotton(Gossypium hirsutum L.). Crop Journal. 2015;3(4):343-52.

59. Schaller F, Biesgen C, Müssig C, Altmann T, Weiler EW. 12-Oxophytodienoate reductase 3 (OPR3) is the isoenzyme involved in jasmonate biosynthesis. Planta. 2000;210(6):979-84 
60. Jie W, Ge X, Li F, Zhang C. Expression profile analysis and functional predictions of the AOS gene family in somatic embryos of upland cotton. Cotton Science. 2016;28(6):538-46.

61. Kolattukudy PE. Polyesters in higher plants. Adv Biochem Eng Biotechnol. 2001;71:1.

62. Kosma DK, Jenks MA. Eco-physiological and molecular-genetic determinants of plant cuticle function in drought and salt stress tolerance. In: Jenks MA, Hasegawa PM, Jain SM, editors. Advances in molecular breeding toward Drought \& Salt Tolerant Crops. Dordrecht, The Netherlands: Springer; 2007. p. 91-120.

63. Zhang X, Henriques R, Lin SS, Niu QW, Chua NH. Agrobacterium-mediated transformation of Arabidopsis thaliana using the floral dip method. Nat Protoc. 2006;1 (2):641-6.

64. Yin R, Skvortsova MY, Loubéry S, UIm R. COP1 is required for UV-B-induced nuclear accumulation of the UVR8 photoreceptor. Proc Natl Acad Sci U S A. 2016;113(30):E4415.

65. Li X, Pei Y, Sun Y, Liu N, Wang P, Liu D, et al. A cotton Cyclin-dependent kinase $\mathrm{E}$ confers resistance to Verticillium dahliae mediated by Jasmonateresponsive pathway. Front Plant Sci. 2018;9:642.

66. Liu Y, Schiff M, Marathe R, Dinesh-Kumar SP. Tobacco Rar1, EDS1 and NPR1/ NIM1 like genes are required for $\mathrm{N}$-mediated resistance to tobacco mosaic virus. Plant J. 2002:30(4):415-29.

67. Liu N, Zhang X, Sun Y, Wang P, Li X, Pei Y, et al. Molecular evidence for the involvement of a polygalacturonase-inhibiting protein, GhPGIP1, in enhanced resistance to Verticillium and Fusarium wilts in cotton. Sci Rep. 2017;7:39840.

\section{Publisher's Note}

Springer Nature remains neutral with regard to jurisdictional claims in published maps and institutional affiliations.

Ready to submit your research? Choose BMC and benefit from:

- fast, convenient online submission

- thorough peer review by experienced researchers in your field

- rapid publication on acceptance

- support for research data, including large and complex data types

- gold Open Access which fosters wider collaboration and increased citations

- maximum visibility for your research: over $100 \mathrm{M}$ website views per year

At BMC, research is always in progress.

Learn more biomedcentral.com/submissions 